\title{
Estimation of Optimal Channel Gain in Cognitive Radio Networks Using Bisectional Algorithm
}

\author{
Kayalvizhi E \\ Post Graduate, Department of Electronics Engineering, MIT Campus, Anna University, Chennai, India \\ Email: kayalvizhi179@gmail.com \\ Balamurugan Gopalakrishnan \\ Assistant Professor, Department of Electronics Engineering, MIT Campus, Anna University, Chennai, India \\ Email: balanmail12@gmail.com
}

\begin{abstract}
In Cognitive radio network, to carry out spectrum sharing between the primary users and cognitive users the interference temperature must be known. When Cognitive Transmitter (CT) knows the interference temperature, it will be able to share the spectrum along with the Primary Transmitter (PT) without affecting the quality of service of the primary users. So, to determine interference temperature from the cognitive transmitter, the primary channel gain must be calculated from the CT. To calculate the primary channel gain a maximum likelihood estimator (MLE) has been designed. In order to reduce the complexity of the maximum likelihood (ML) estimator, a Bisectional algorithm has been introduced to estimate the optimal channel gain from CT. The estimation error is very less of about $\mathbf{- 0 . 8 8}$ and it is reduced to $\mathbf{- 0 . 9 2}$ as the number of block increases to 100 . The channel gain approaches to true value, when the number of blocks increases to 100 . The achieved rate of $\mathrm{CT}$ is 18 kbps and the estimator approaches to true value as $L$ block increases with perfect primary channel gain.
\end{abstract}

Keywords - Primary channel gain, MLE, Interference temperature, Spectrum sharing.

\section{INTRODUCTION}

The development in wireless communication has made the spectrum as a scarce resource $[1,2]$. Traditionally, the unlicensed users are not allowed to access the spectrum even when it is not been utilized. This empowers the development of cognitive radio, which allows the unlicensed users to access the licensed bands but keeping the interference to the primary users at a low level and this could meet the stringent requirements in wireless communication [3].

The cognitive users can utilizes the licensed spectrum allocated to the primary users by two types of spectrum access techniques. By using the spectrum access techniques the cognitive users can utilizes the spectrum for transmission without affecting the licensed users. The spectrum access mechanism has been classified as opportunistic spectrum access [4] and spectrum sharing technique[5]. In the opportunistic spectrum access technique, the SU can access the channel only when PU is not utilizing the channel [6]. In spectrum sharing access technique, the SU can share the spectrum along with the PU by transmitting below the interference temperature. Since interference temperature plays a crucial role to control the transmit power and protect primary transmissions [7]. Therefore, compared with the opportunistic spectrum access, spectrum sharing is able to exploit more spectrum opportunities, obtain higher spectrum utilization efficiency, and thus achieve higher cognitive throughput. [8] an underlay cognitive radio network, the cross channel gain from a cognitive transmitter $(\mathrm{CT})$ to a primary receiver $(\mathrm{PR})$ is crucial for spectrum sharing.

In this paper, the primary transmitter (PT) transmits the signal to the primary receiver (PR). If the signal being received at the PR didn't match the target signal to noise ratio (SNR) then the PT automatically increase its transmit power to satisfy the target SNR at the PR. When the primary channel gain is large, then even when the PT transmits at low power, the target SNR could be reached at the PR. When the primary channel gain is small, then the PT increase the transmit power to satisfy the target SNR at the PR [7].

If the primary channel gain is high, the interference temperature is calculated based on channel gain and thus the interference temperature will also be high that cognitive user can transmit at high power thus throughput can be increased. If the CT want to share the spectrum along with PT then CT must know the interference temperature. The primary channel gain must be known by the CT to calculate the interference temperature $[9,10]$.To obtain the primary channel gain, CT needs a link from the primary system, thus the primary system can send the primary channel gain to $\mathrm{CT}$. But in practice, this link between primary and cognitive system is not possible [11]. To overcome this issue a method for estimation of channel gain from CT has been proposed in this paper. Thus a Maximum likelihood estimator has been designed to estimate the primary channel gain with high accuracy from CT. The CT calculates the interference temperature from estimated channel gain and carries out spectrum sharing. 
The paper is organized as follows: Section II describes the system model to carry out spectrum sharing between primary and cognitive users. Section III presents the design of Maximum likelihood estimator. In section IV the bisectional algorithm to reduce the complexity of $\mathrm{ML}$ estimator is explained. Section V describes interference temperature. In Section VI, presents the performance analyses of proposed maximum likelihood estimator to estimate the channel gain and carry out spectrum sharing. Finally, conclusions are drawn in Section VII.

\section{SySTEM MODEL}

The system model is shown in the Fig 1 consists of PT, CT, PR, and CR. The spectrum sharing is to be carried out between the PT and CT. Initially, the primary signal from PT has been transmitted with unit power and it is received at the PR is detected which would be affected by the fading and noise of the transmitted channel.

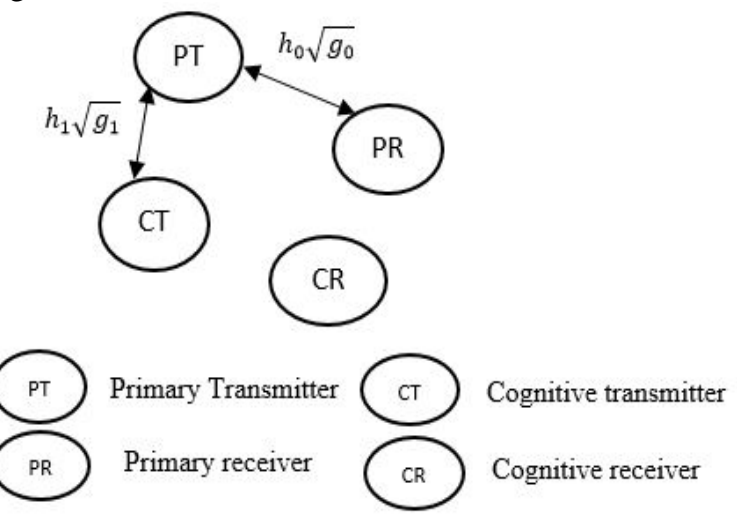

Figure 1. System model for carrying out spectrum sharing between primary and cognitive users.

In this system model, PT transmits signal to the PR on certain frequency band. The $\mathrm{CT}$ also transmit at the same frequency band and realize on spectrum sharing. Thus, PT should have the knowledge about the primary channel gain and the transmitted signal from PT should contains some information about the primary channel gain.

\section{Channel model:}

The channel between the PT and the PR is $h_{0} \sqrt{g_{0}}$ and the channel between the PT and CT is $h_{1} \sqrt{g_{1}}$. Where $h_{j}$ the fading follows Rayleigh distribution and it remains constant within a block and varies among different block.

The channel gain between the transceivers is determined using the path-loss model [12]

$$
P_{l}\left(d_{j}\right)=128+37.6 \log _{10}\left(d_{j}\right) \text { for } d_{j} \geq 0.035 \mathrm{~km}
$$

Where $d_{j}(\mathrm{~km})$ is distance between two transceiver, the large-scale channel gain $g_{j}$ is expressed as,

$$
g_{j}=10^{-12.8} d_{j}^{-3.76} \quad \text { for } d_{j} \geq 0.035 \mathrm{~km}
$$

\section{Signal model:}

The PT transmits a primary signal to PR. The PR must satisfy the target SNR. If the target SNR is not satisfied, then it increases the transmits power to satisfy the target SNR. The CT estimates the primary channel gain by sensing the primary signal.

\section{Signal model from PT to PR:}

The primary signal has been transmitted from PT is $x_{\text {pri }}$. Initially, the input signal is transmitted with unit power $\mathrm{p}_{0}$. The received signal at the PR for L blocks is expressed as,

$$
y_{p r i}(l)=h_{0}(l) \sqrt{g_{0} p_{0}(l)} x_{p r i}(l)+n_{p r i}(l)
$$

where $n_{p r i}$ is the additive white Gaussian noise (AWGN) at the primary receiver with unit mean and variance $\sigma^{2}$.

The SNR of the received signal at PR is given as

$$
\gamma_{p r i}(l)=\frac{\left|h_{0}(l)\right|^{2} g_{0} p_{0}(l)}{\sigma^{2}}
$$

If the target SNR at PR is not satisfied, then the transmitter power at PT has to be increased for the $\mathrm{L}$ blocks as

$$
p_{p r i}(l)=\frac{\gamma_{T a r} \sigma^{2}}{\left|h_{0}(l)\right|^{2} g_{0}}
$$

\section{Signal model from PT to CT:}

The received signal for $\mathrm{L}$ blocks at the $\mathrm{CT}$ is measured as

$$
y_{c o g}(l)=h_{1}(l) \sqrt{g_{1} p_{0}(l)} x_{p r i}(l)+n_{c o g}(l)
$$

The SNR of the received signal at CT is measured as

$$
\gamma_{c o g}(l)=\frac{\left|h_{1}(l)\right|^{2} g_{1} p_{0}(l)}{\sigma^{2}}
$$

Substitute (5) in the equation $\gamma_{c o g}(l)$ we get

$$
\gamma_{c o g}(l)=\frac{\gamma_{T a r} g_{1}\left|h_{1}(l)\right|^{2}}{g_{0}\left|h_{0}(l)\right|^{2}}
$$

\section{DESIGN OF MAXIMUM LIKELIHOOD ESTIMATOR}

Maximum likelihood estimator has been designed to estimate the primary channel gain between the PT and PR from the CT. The basic principle followed by the estimator design is described below:

\subsection{Basic principle:}

The ML estimator establishes the decision based on the received signal samples. Likelihood is defined as the conditional PDF of the received signal samples. No prior information is known about the actual channel gain. So, from the obtained $K$ samples the most detected samples 
which will have equal likeliness is considered. Finally, log function is taken to likelihood, because the log likelihood function is maximum the estimated value will be closer to the actual value.

As the number of samples detected for estimation of the primary channel gain increases, it converges to true value. This property makes the ML estimator more consistent. The primary signal has been transmitted from PT contains information about the primary channel gain and thus the ML estimator can estimate the channel gain by sensing the primary signal has been transmitted from the PT.

\subsection{Estimator design:}

The ML estimator estimates the channel gain without any prior knowledge of the channel gain. It finds the channel gain only from the estimated samples in $L$ block. By removing the block index $L$ from the equation (7), the measured SNR at CT is expressed as

$$
\gamma_{\operatorname{cog}}=\frac{\gamma_{\operatorname{Tar}} g_{1} \emptyset}{g_{0}}
$$

where $\varnothing=\frac{\left|h_{1}(l)\right|^{2}}{\left|h_{0}(l)\right|^{2}}$

The cumulative distribution function (CDF) of the SNR measured at the CT is expressed in equation 9 ,

$$
\begin{aligned}
F_{\Gamma_{C}}\left(\gamma_{C o g}\right) & =p_{r}\left\{\frac{g_{1} \gamma_{T a r} \emptyset}{g_{0}} \leq \gamma_{\operatorname{cog}}\right\} \\
& =p_{r}\left\{\varnothing \leq \frac{g_{0} \gamma_{\operatorname{cog}}}{g_{1} \gamma_{T a r}}\right\} \\
& =F_{\varnothing}\left(\frac{g_{0} \gamma_{\operatorname{cog}}}{g_{1} \gamma_{T a r}}\right)
\end{aligned}
$$

where $F_{\emptyset}($.$) denotes the CDF of \varnothing$

The CDF of $\emptyset$ is represented in equation 10 ,

$$
F_{\varnothing}(\varnothing)=\frac{\emptyset}{1+\emptyset}
$$

After substituting equation 10 in 9 , we have $\mathrm{CDF}$ of $\gamma_{\operatorname{Cog}}$ as

$$
F_{\Gamma_{\operatorname{Cog}}}\left(\gamma_{\operatorname{Cog}}\right)=\frac{g_{0} \gamma_{\operatorname{cog}}}{g_{1} \gamma_{T a r}+g_{0} \gamma_{\operatorname{cog}}}
$$

we have to take the derivative of the cumulative distribution function (CDF) to find the probability density function (PDF) of $\gamma_{\operatorname{Cog}}$ is

$$
f_{\gamma_{\operatorname{Cog}}}\left(\gamma_{\operatorname{Cog}}\right)=\frac{g_{0} \gamma_{T} g_{1}}{\left(g_{1} \gamma_{T a r}+g_{0} \gamma_{\operatorname{cog}}\right)^{2}}
$$

The fading caused by Rayleigh distribution $h_{0}$ and $h_{1}$ varies independently among the different blocks. The relation of $h_{0}$ and $h_{l}$ in $\gamma_{\operatorname{Cog}}$ varies independently among the $L$ consecutive samples. Thus the joint PDF of $\gamma_{\operatorname{Cog}}$ for the $L$ consecutive blocks is expressed as,

$$
f_{\gamma_{\operatorname{Cog}}}\left(\gamma_{\operatorname{Cog}}(1), \gamma_{\operatorname{Cog}}(2) \ldots \gamma_{\operatorname{Cog}}(l)\right)=\prod_{l=1}^{L} \frac{g_{0} \gamma_{\operatorname{Tar}} g_{1}}{\left(g_{1} \gamma_{T a r}+g_{0} \gamma_{\operatorname{cog}}(l)\right)^{2}}
$$

To find the optimal $g_{0}{ }^{*}$, the joint PDF should be maximized by taking log on both sides in equation 13 . The $\log$ function is taken to Max likelihood estimator, because it estimates the value closer to the actual value and it also makes the equation simpler.

$$
\begin{aligned}
& \log f_{\gamma_{\operatorname{Cog}}}\left(\gamma_{\operatorname{Cog}}(1), \gamma_{\operatorname{Cog}}(2) \ldots \gamma_{\operatorname{Cog}}(l)\right)= \\
& \sum_{l=1}^{L} \log g_{0} 2 \log \left[g_{1} \gamma_{T a r}+g_{0} \gamma_{\operatorname{cog}}(l)\right]
\end{aligned}
$$

If we denote $\overline{g_{0}}=\log g_{0}$ we can rewrite the equation (14) as

$$
\begin{aligned}
& \log f_{\gamma_{\operatorname{Cog}}}\left(\gamma_{\operatorname{Cog}}(1), \gamma_{\operatorname{Cog}}(2) \ldots \ldots \gamma_{\operatorname{Cog}}(l)\right)= \\
& \sum_{l=1}^{L} \overline{g_{0}}-2 \log \left[g_{1} \gamma_{\text {Tar }}+e^{\bar{g}_{0}} \gamma_{\operatorname{cog}}(l)\right] \triangleq f_{1}\left(\overline{g_{0}}\right)
\end{aligned}
$$

$f_{1}\left(\overline{g_{0}}\right)$ is the concave function of $\overline{g_{0}}$, to check the concavity it should be proved that $f_{1}{ }^{\prime \prime}\left(\overline{g_{0}}\right) \leq 0$

To begin with, we considered the first order derivative of $f_{1}\left(\overline{g_{0}}\right)$ in terms $\overline{g_{0}}$ as

$$
f_{1}^{\prime}\left(\overline{g_{0}}\right)=\quad \sum_{l=1}^{L} 1-\frac{2 e^{\bar{g}_{0}} \gamma_{\operatorname{cog}}(l)}{g_{1} \gamma_{T a r}+e^{\bar{g}_{0}} \gamma_{\operatorname{cog}}(l)}
$$

Then the second order derivative of $f_{1}\left(\overline{g_{0}}\right)$ is considered as,

$$
f_{1}^{\prime \prime}\left(\overline{g_{0}}\right)=\quad \sum_{l=1}^{L}-\frac{2 e^{\bar{g}_{0}} \gamma_{\operatorname{cog}}(l) g_{1} \gamma_{T a r}}{\left[g_{1} \gamma_{T a r}+e^{\bar{g}_{0}} \gamma_{\operatorname{cog}}(l)\right]^{2}}
$$

If the above equation (17) is less than zero i.e. $f_{1}^{\prime \prime}\left(\overline{g_{0}}\right) \leq$ 0 , then the concavity is proved and we can estimate the optimal channel gain from the function of $g_{0}$.

The estimation error of the ML estimator is determined by,

$$
\text { Error }=\left|\frac{10 \log _{10}\left(\widehat{g_{0}}\right)-10 \log _{10}\left(g_{0}\right)}{10 \log _{10}\left(g_{0}\right)}\right|
$$

where $\widehat{g_{0}}$ is the estimated primary channel gain and $g_{0}$ is the actual channel gain between the primary transceivers.

\section{BISECTIONAL ALGORITHM}

The bisectional algorithm is to reduce the computational complexity of the ML estimator. The detailed bisectional algorithm is shown in the Fig 2. In the bisectional algorithm $g_{0}^{\min }$ and $g_{0}^{\max }$ are taken as the initial values from the estimated primary channel gain in $L$ blocks by the ML estimator.

The bisectional algorithm is also called as intermediate value method. In this method the optimal channel gain can be estimated, when the estimated channel gain is less than the error tolerance. The error tolerance of bisectional algorithm is set as a default value $1.5 \times 10^{-6}$ because in this value it provides a good performance across a broad range of problems. 


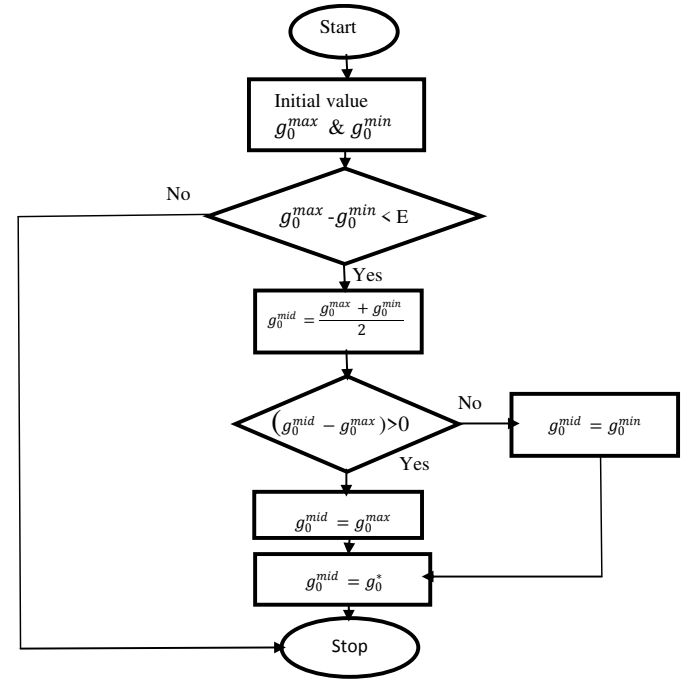

Figure 2. Bisectional Algorithm

When the estimated primary channel gain is less than the error tolerance then the intermediate value is found to estimate the optimal channel gain. When the channel gain is less than the threshold value, then $g_{0}^{\min }$ is selected as the optimal channel gain otherwise $g_{0}^{\max }$ is selected as the optimal channel gain.

\section{INTERFERENCE TEMPERATURE}

The interference temperature is defined as the temperature equivalent to the RF power available at a receiving antenna per unit bandwidth [13]. The CT estimates the optimal channel gain with the help of the bisectional algorithm. The interference temperature could be calculated from the estimated optimal channel gain and spectrum sharing could be realized.

When PT is transmitting data to PR the target SNR must be matched, if the target SNR is not matched the PT is transmitting at the increased power to satisfy the target SNR. Even when the PT is transmitting at the maximum power if the target SNR at PR is not matched then it is due to the interference from the cognitive transmission, at this situation outage probability of the primary signal is claimed. Here the outage probability is assumed as $\theta=0.01$ [14]. Thus the interference temperature imposed on the transmit power of the CT to protect the primary transmission is given as

$$
\text { interference temp }=\frac{-p_{\max } g_{0} \ln (1-\theta)}{\gamma_{T a r}}-\sigma^{2}
$$

Where $\mathrm{g}_{0}$ is the large-scale channel gain between the primary transceivers, $p_{\max }$ is the maximum transmit power of the PT and $\sigma_{2}$ represents the power of the AWGN.

\section{Simulation ReSUlt}

In this section we have shown the simulation results to evaluate the performance of the proposed maximum likelihood estimator to estimate the channel gain and carry out spectrum sharing. We have adopted a system model as discussed in section 2, where the coverage radius of the PT is $\mathrm{R}=0.5 \mathrm{~km}$ shown in Fig 3 . The coordinate of PT, PR, CT and $\mathrm{CR}$ is set as $(0,0),(0.025 \mathrm{~km}, 0),(0.125 \mathrm{~km}$, $0.2165 \mathrm{~km})$ and $(0.175 \mathrm{~km}, 0.2105)$ respectively. The target $\mathrm{SNR}$ at the PR is fixed as $\gamma_{T a r}=10$. Initially the transmit power of the signal from PT is unit power and if the target SNR is not been satisfied at PR then the transmit power could be increased further. We assumed the power of the AWGN is $-114 \mathrm{dBm}$.

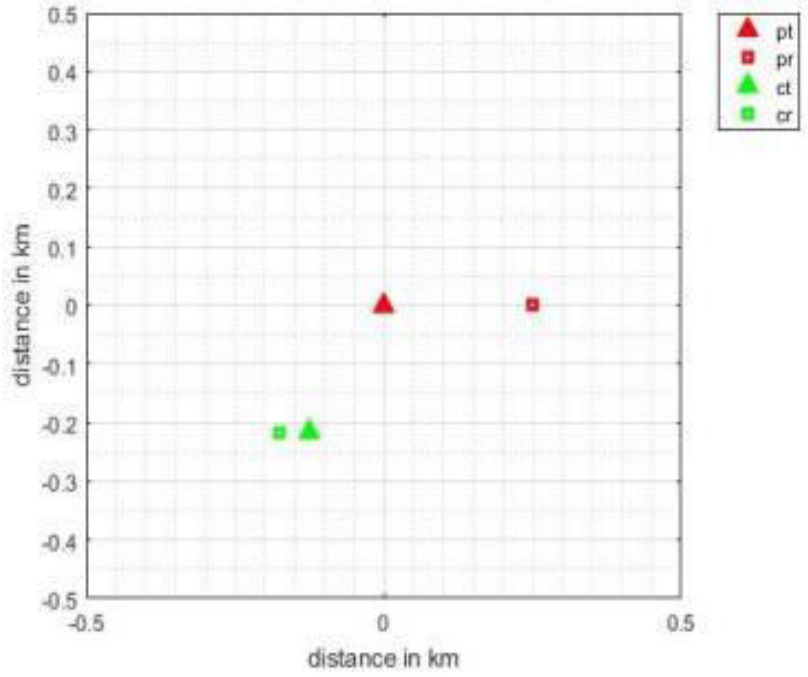

Figure 3. Simulation scenario

\subsection{Estimation of primary channel gain:}

The maximum likelihood estimator, estimates the primary channel gain for several blocks and estimates the optimal channel gain. From the obtained $L$ blocks of samples, the most detected symbols which have equal likeliness is considered for estimation in ML estimator. As the number of samples detected for estimation increases, the channel gain converges to true value which is shown in Fig 4.

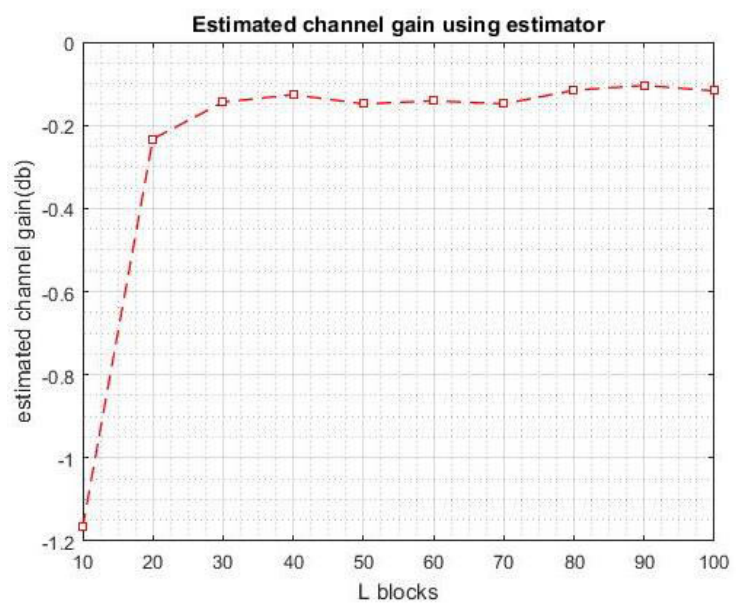

Figure 4. Estimation of channel gain

The estimation of channel gain is $-1.22 \mathrm{db}$ for 10 blocks and when the number of blocks increases it approaches to about $-0.7 \mathrm{db}$. As the number of block increases, the channel gain approaches to true value. The ML estimator 
has an feature that it can estimate the gain without any prior knowledge of the primary channel gain.

\subsection{Estimation Error:}

Fig 5 shows the estimation error of the ML estimator. The estimation error is determined to estimate the primary channel gain from the CT used to carry the spectrum sharing. When the number of the block $(L)$ is increased from 10 to 100 the estimation error starts decreases from $0.88 \mathrm{db}$ to $-0.92 \mathrm{db}$.

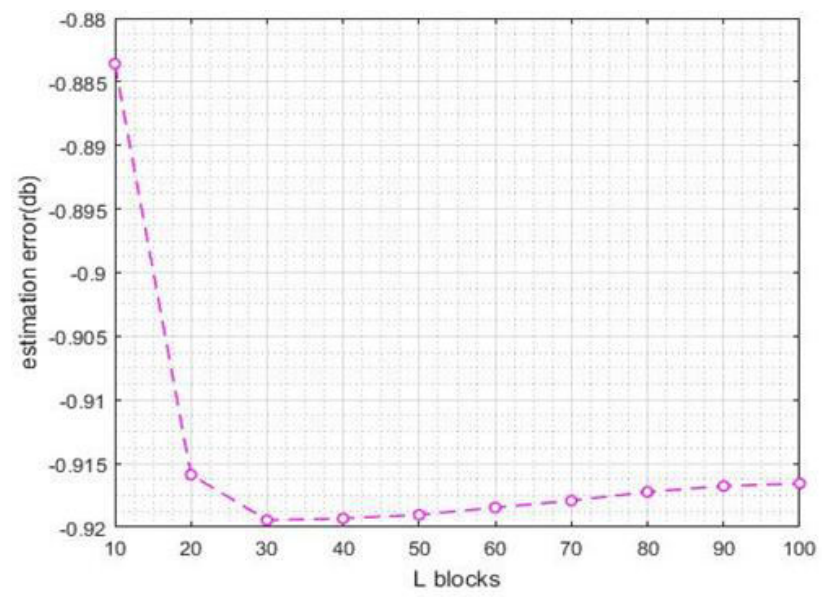

Figure 5. Estimation error

The estimation error is reduced in the ML estimator because the ML estimator estimates the channel gain with more accurate as the number of block $L$ increases. The estimation error is reduced because the estimated value approaches to true value when the number of block increases to 100 .

\subsection{Computational Complexity:}

Fig 6 shows the average time to estimate primary channel gain from CT by the ML estimator. The computational complexity for the designed ML estimator is less because the required time for estimation is very less from $(0.1-0.2)$ second irrespective to increase in block size. From the result it concludes that ML estimator shows lesser computational complexity.

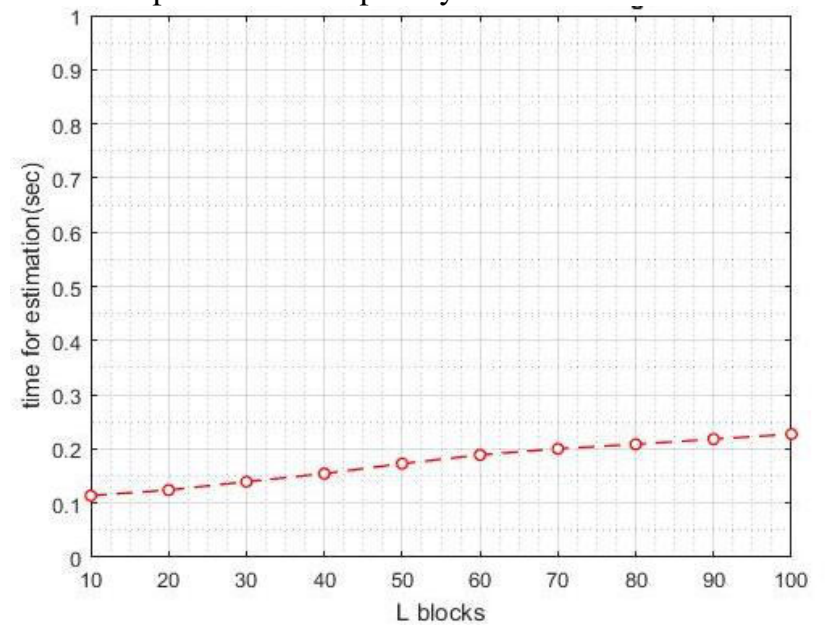

Figure 6. Estimation of computational complexity

\subsection{Achievable rates:}

The achievable rate of CT, by estimating ML using bisectional algorithm by incresing the block $L$ is shown in Fig 7. The acutal achievable rate is upto $18 \mathrm{kbps}$ and when the number of blocks increased the estimated rate reaches close to the actual rate. Thus, the primary channel gain estimated using the ML estimator using the bisectional algorithm has been presented and the optimal channel gain closer to the actual channel gain is estimated.

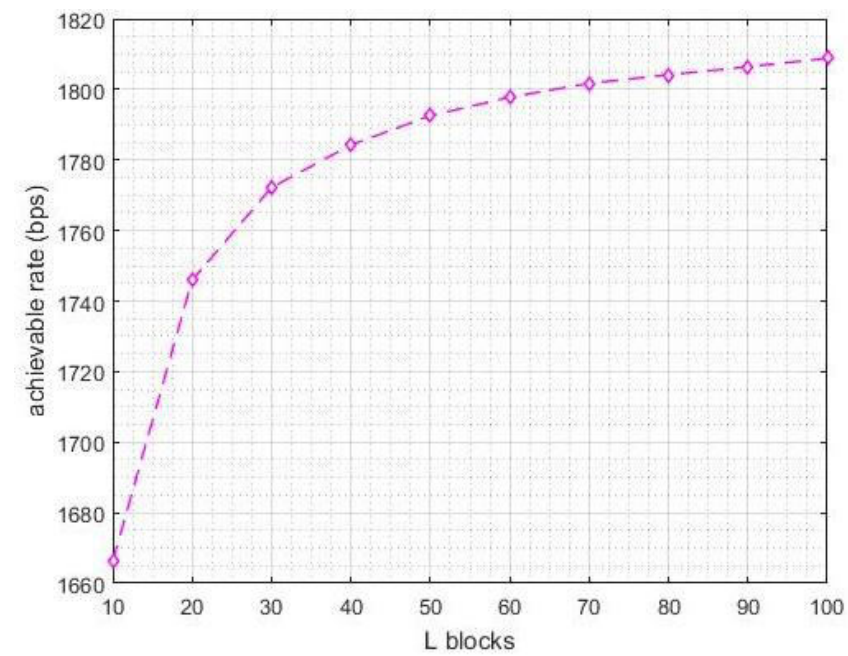

Figure 7. Estimated achievable rate at CT

\section{CONCLUSION}

In this paper, the proposed method sense the primary signal for $L$ blocks to estimate the primary channel gain and thus CT will be able to calculate the interference temperature of the primary system to carry out spectrum sharing. The ML estimator, estimates the channel gain with more accuracy, the estimated channel gain approaches to true value when the block increases from 10 to 100. The proper selection of block is necessary to balance the system complexity and estimation error. The estimation error is reduced from -0.88 to -0.92 by increasing the number of blocks.

\section{REFERENCES}

[1] N. Devroye, P. Mitran and V. Tarokh, Achievable rates in cognitive radio channels, IEEE Trans. Inf. Theory, 52(5), 2006, 1813-1827.

[2] S. Shanmugavel, M. A. Bhagyaveni and R. Kalidoss, Cognitive Radio-An Enabler for Internet of Things, Delft, The Netherlands:River Publishers, 2017.

[3] J. Mitola, III, Software radio architecture: A mathematical perspective, IEEE J. Sel. Areas Commun., 17(4), 2010, 514-538.

[4] S. Huang, X. Liu and Z. Ding, Opportunistic spectrum access in cognitive radio networks, Proc. 27th Conf. Comput. Commun. (IEEE INFOCOM 2008), 2008, 1427-1435. 
[5] S. Pandit, and G. Singh, An overview of spectrum sharing techniques in cognitive radio communication system, Wireless Netw. 23(2), 2017, 497-518.

[6] Q. Zhao and BM. Sandler, A survey of dynamic spectrum access, IEEE Signal Process. Mag., 24(3), 2007, 79-89.

[7] A. Ghasemi and ES. Sousa, Fundamental limits of spectrum-sharing in fading environments, IEEE Trans. Wireless Commun., 6(2), 2007, 649-658.

[8] L. Zhang, G. Wu, G. Zhao, Y. Liang and S. Li, Proactive Cross-Channel Gain Estimation for Spectrum Sharing in Cognitive Radio, IEEE journal on selected areas in communications, 34(10) , 2016, 2776-2790.

[9] EA. Jorswieck, L. Badia, T. Fahldieck, E. Karipidis, and J. Luo, Spectrum sharing improves the network efficiency for cellular operators, IEEE Commun. Mag., 52(3), 2014, 129-136.

[10] A. Arthy and P. Periyasamy, A Review on Spectrum Sensing Techniques in Cognitive Radio Network, International Journal of Advanced Networking and Applications (IJANA), 2015, 80-83.

[11]L. Zhang, G. Zhao, G. Wu, and Z. Chen, Proactive channel gain estimation for coexistence between cognitive and primary users, in Proc. IEEE Global Commun. Conf. (GLOBLECOM), Anaheim, CA, USA, 2012, pp. 1-6.

[12] Physical Layer Aspects for Evolved Universal Terrestrial RadioAccess (UTRA), document 3GPP TR 25.814, 3GPP, 2006.

[13] A. M. Wyglinski, M. Nekovee and T. Hou, Cognitive Radio Communication and Networks, in Academic Press, 2010.

[14] L. Zhang, G. Zhao, W. Zhou, L. Li, G. Wu, Y. Liang, and S. Li, Primary Channel Gain Estimation for Spectrum Sharing in Cognitive Radio Networks, IEEE transactions on communications, 65(10), 2016, 4152-4162. 